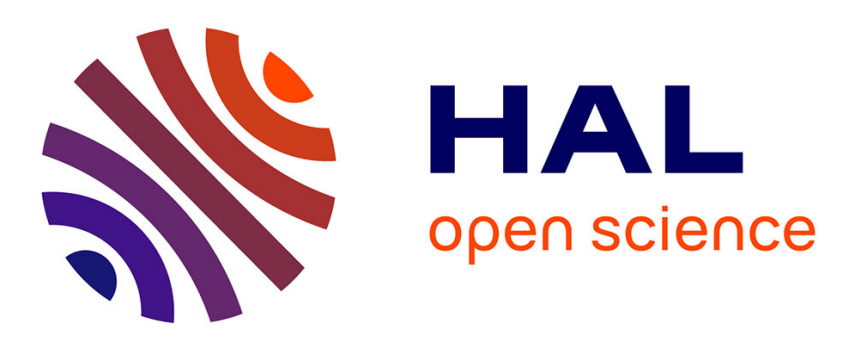

\title{
Etude systématique de la commande d'une alimentation pulsée pour systèmes magnétiques de forte puissance S. Allano, C. Glaize
}

\section{To cite this version:}

S. Allano, C. Glaize. Etude systématique de la commande d'une alimentation pulsée pour systèmes magnétiques de forte puissance. Revue de Physique Appliquée, 1982, 17 (1), pp.37-44. 10.1051/rphysap:0198200170103700 . jpa-00244967

\section{HAL Id: jpa-00244967 https://hal.science/jpa-00244967}

Submitted on 1 Jan 1982

HAL is a multi-disciplinary open access archive for the deposit and dissemination of scientific research documents, whether they are published or not. The documents may come from teaching and research institutions in France or abroad, or from public or private research centers.
L'archive ouverte pluridisciplinaire HAL, est destinée au dépôt et à la diffusion de documents scientifiques de niveau recherche, publiés ou non, émanant des établissements d'enseignement et de recherche français ou étrangers, des laboratoires publics ou privés. 
Classification

Physics Abstracts

07.50

\title{
Etude systématique de la commande d'une alimentation pulsée pour systèmes magnétiques de forte puissance
}

\author{
S. Allano et C. Glaize \\ Ecole Normale Supérieure de l'Enseignement Technique \\ Laboratoire de Physique Appliquée, 61, avenue du Président-Wilson, 94230 Cachan, France
}

(Reçu le 3 juillet 1981, révisé le 8 octobre 1981, accepté le 12 octobre 1981)

\begin{abstract}
Résumé. - Une grande part des performances obtenues avec une machine à réluctance variable provient des caractéristiques de son alimentation. Cela nous a amené à introduire un convertisseur original pour l'alimentation de systèmes magnétiques fortement inductifs. La recherche de performances accrues avec en particulier une haute sûreté de fonctionnement nous a conduit à étudier de manière plus approfondie les possibilités de ce convertisseur. Nous décrivons toutes les phases de fonctionnement possibles, ce qui permet de prévoir l'aboutissement de toute séquence normale ou anormale. L'étude tout à fait théorique du convertisseur est complétée par une expérimentation sur une machine à réluctance variable.
\end{abstract}

\begin{abstract}
A large part of the results obtained with a reluctance machine arise from the characteristics of its supply. This led us to introduce an original converter for the supply of highly inductive magnetic systems. The study of results achieved with in particular a high level of operative reliability led us to consider in greater depth this converter's possibilities. We shall describe all the possible operationnal phases, which will permite us to foresee the end-result of any normal or abnormal sequence. This entirely theorical study is completed by an experiment with a high-power reluctance machine.
\end{abstract}

Pour l'alimentation d'enroulement de systèmes électrotechniques présentant une forte inductance, nous avons été amenés à concevoir un convertisseur (Fig. 1) permettant d'injecter avec un bon rendement une puissance de l'ordre de quelques kilowatts à quelques dizaines de kilowatts sous la forme d'un courant se rapprochant le plus possible d'un créneau. Les deux applications principales étudiées sont les alimentations d'un électro-aimant et d'une machine à réluctance variable monophasée non excitée. Le convertisseur a pour rôle de brancher successivement

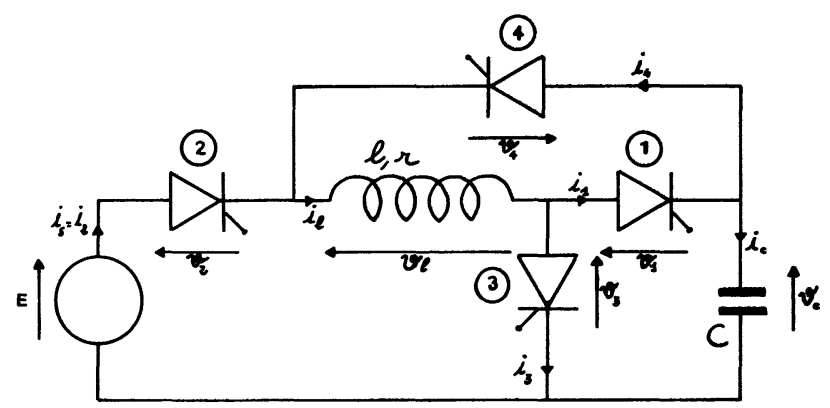

Fig. 1. - Convertisseur d'alimentation.

[Power converter.] le bobinage aux bornes d'une source de tension (pour l'alimentation) et/ou d'un condensateur (pour les montées et descentes rapides du courant par transfert d'énergie). Nous avons déjà introduit et utilisé ([1], [2]) un convertisseur et une commande simple conçus pour cet usage.

Afin d'exploiter au mieux ses possibilités ainsi que pour accroître la fiabilité de l'ensemble, il est nécessaire d'étudier toutes les phases de fonctionnement du convertisseur et les transitions possibles et interdites entre elles.

1. Etude des phases de fonctionnement. - -1.1 REMARQUies PRÉlIMINAIRES. - La présence des thyristors impose au courant dans le bobinage d'être toujours positif ou nul $\left(i_{l} \geqslant 0\right)$.

La présence du thyristor 2 impose au courant d'alimentation d'être toujours positif ou nul. Si l'on désire que la source d'alimentation puisse travailler en récupération, il est alors nécessaire d'inverser son signe. Dans ce qui suit, on étudiera principalement le cas $E>0$. Le cas $E \leqslant 0$ est décrit dans [3].

On peut aussi noter que toutes les commutations sont provoquées (puisqu'il n'y a aucune diode dans le montage) sauf dans le cas où $i_{l}$ s'annule. On a 
alors dans ce dernier cas seulement une extinction naturelle de tous les thyristors.

Les thyristors 1 et 3 peuvent être considérés comme un inverseur commandé. La commande (envoi d'une impulsion sur le thyristor concerné) ne pourra agir que si une condition sur $v_{c}$ est vérifiée :

$$
1 \underset{\text { possible si } v_{c}<0}{\stackrel{\text { possible si } v_{c}>0}{\rightleftharpoons}} 3 \text {. }
$$

Le cas $v_{c}=0$ sera examiné plus tard.

Il en est de même pour les thyristors 2 et 4 :

$$
2 \underset{\text { possible si } v_{c}<E}{\stackrel{\text { possible si } v_{c}>E}{\rightleftharpoons}} 4 .
$$

Toutes les phases de fonctionnement sont représentées par le(s) numéro(s) des thyristors conducteurs. La phase (0) correspond à la non-conduction de tous les thyristors. Pour chaque phase, on donne :

- le schéma partiel de fonctionnement,

- les équations différentielles d'évolution,

- les courants et tensions dans et aux bornes des thyristors,

\section{- ses propriétés.}

2.2 Etude De Chaque Phase. - Phase (0). C'est la phase où aucun thyristor ne conduit. On y arrive, en extinction naturelle, dès que $i_{l}=0$.

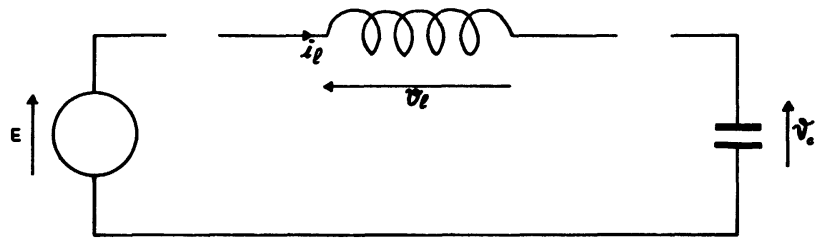

Fig. 2.

$$
i_{l}=0 \Rightarrow \frac{\mathrm{d} \varphi}{\mathrm{d} t}=0
$$

Les courants sont tous nuls.

$$
v_{c}=\mathrm{Cte} \Rightarrow \frac{\mathrm{d} v_{c}}{\mathrm{~d} t}=0 .
$$

La tension aux bornes du condensateur reste constante.

La tension maximale que peut avoir à bloquer un thyristor est $\operatorname{Sup}\left(v_{c}, E-v_{c}\right)$.

- Phases (1), (2), (3), (4). - Il ne peut exister de phases dans laquelle un thyristor seulement soit conducteur.

- Phase (1)-(2). - L'enroulement est branché en série avec la source $E$ et le condensateur $C$. Cette phase est aussi appelée $" E+C »$.

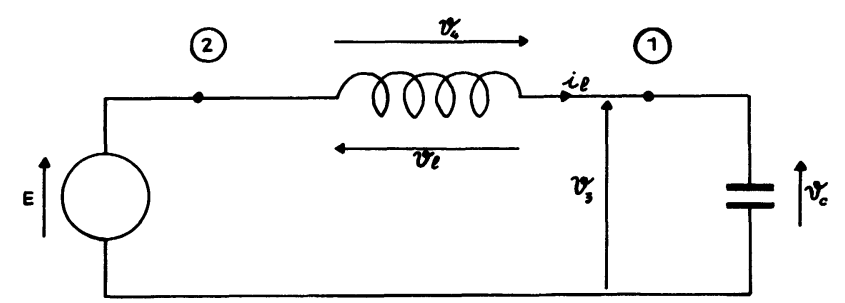

Fig. 3.

$$
v_{l}=\frac{\mathrm{d} \varphi}{\mathrm{d} t}+r_{.} i_{l}=E-v_{c}
$$

$C \cdot \frac{\mathrm{d} v_{c}}{\mathrm{~d} t}=i_{l}(\geqslant 0) \Rightarrow v_{c}$ ne peut que croître.

$$
\begin{array}{llll}
v_{1}=0 & v_{2}=0 & v_{3}=v_{c} & v_{4}=v_{c}-E \\
i_{1}=i_{l} & i_{2}=i_{l} & i_{3}=0 & i_{4}=0 .
\end{array}
$$

La remarque du paragraphe 1.1 montre que l'on ne peut sortir de cette phase que si $v_{c}>\operatorname{Inf}(0, E)$, ou encore, si $i_{l}$ s'annule. Dans ce dernier cas, la tension $v_{c}$ aux bornes du condensateur sera nécessairement supérieure à $E$. En effet, si à un instant donné, $v_{c}<E$, alors $\mathrm{d} \varphi / \mathrm{d} t>0$, le courant $i_{l}$ ne peut s'annuler et $v_{c}$ croît. Si $v_{c}>E$, le courant peut s'annuler et $v_{c}$ ne peut pas diminuer.

- Phase (2)-(3). - La machine est branchée sur la source d'alimentation $E$ («E»).

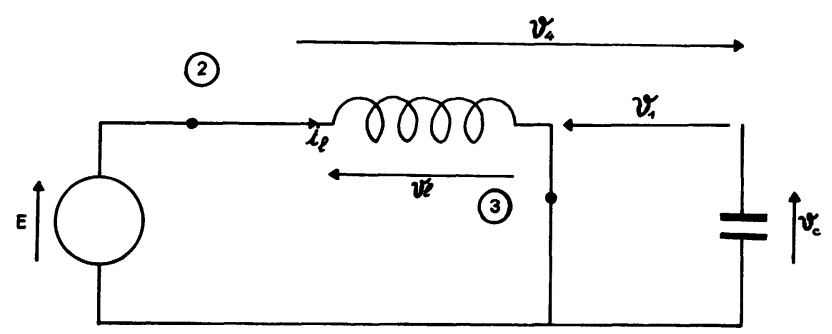

Fig. 4.

$$
v_{l}=\frac{\mathrm{d} \varphi}{\mathrm{d} t}+r i_{l}=E
$$

$$
\frac{\mathrm{d} v_{c}}{\mathrm{~d} t}=0 \quad v_{c} \text { reste constant }
$$

$$
\begin{array}{llll}
v_{1}=-v_{c} & v_{2}=0 & v_{3}=0 & v_{4}=v_{c}-E \\
i_{1}=0 & i_{2}=i_{l} & i_{3}=i_{l} & i_{4}=0 .
\end{array}
$$

On notera que pour $0<v_{c}<E$, il ne peut $\mathrm{y}$ avoir transfert vers une autre phase. Le courant augmente dans l'enroulement, $v_{c}$ reste constant. C'est alors une perte de contrôle irrémédiable.

- Phase (3)-(4) - Le bobinage et le condensateur sont en série $(« C »)$. 
(4)

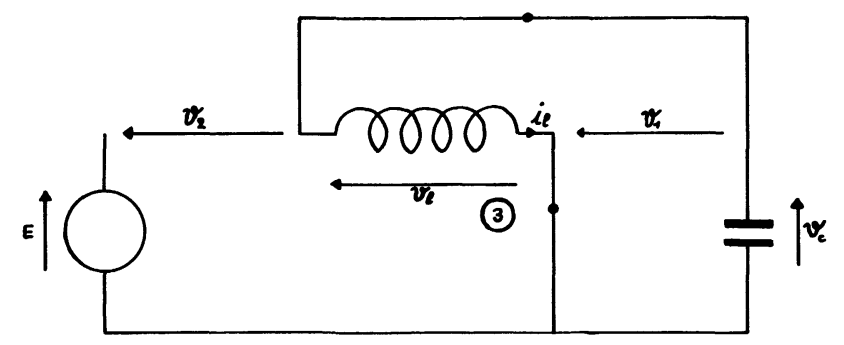

Fig. 5.

$$
\begin{aligned}
v_{l} & =\frac{\mathrm{d} \varphi}{\mathrm{d} t}+r \cdot i_{l}=v_{c} \\
C \cdot \frac{\mathrm{d} v_{c}}{\mathrm{~d} t} & =-i_{l}(<0) .
\end{aligned}
$$

La tension aux bornes du condensateur ne peut que décroître.

$$
\begin{array}{llll}
v_{1}=-v_{c} & v_{2}=E-v_{c} & v_{3}=0 & v_{4}=0 \\
i_{1}=0 & i_{2}=0 & i_{3}=i_{l} & i_{4}=i_{l} .
\end{array}
$$

On peut faire ici les mêmes remarques que pour la phase (1)-(2): on ne peut sortir que $\operatorname{si} v_{c} \leqslant \operatorname{Sup}(0, E)$, soit ici $v_{c} \leqslant 0$ ou encore quand $i_{l}$ s'annule. Dans ce dernier cas, $v_{c}$ sera alors nécessairement inférieure à 0 .

- Phase (1)-(4). - Le bobinage est court-circuité sur lui-même. On n'y apporte ni enlève d'énergie sous forme électrique. Cette phase est nommée phase de roue libre («R.L.»).

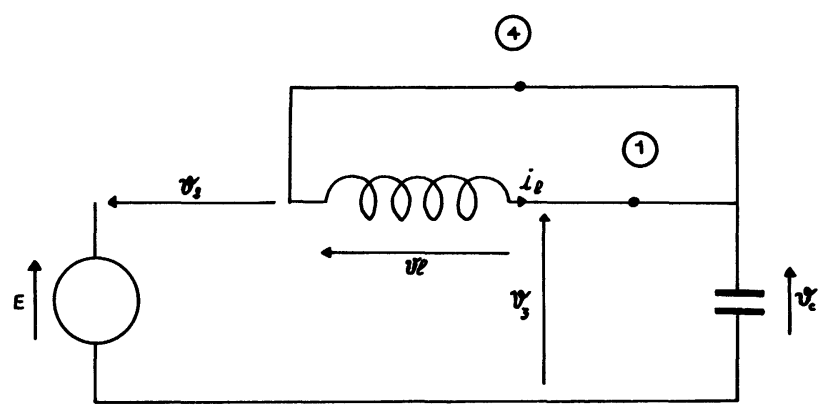

Fig. 6.

$$
\begin{aligned}
v_{l} & =\frac{\mathrm{d} \varphi}{\mathrm{d} t}+r . i_{l}=0 \\
\frac{\mathrm{d} v_{c}}{\mathrm{~d} t} & =0
\end{aligned}
$$

La tension aux bornes du condensateur est constante.

$$
\begin{array}{llll}
v_{1}=0 & v_{2}=E-v_{c} & v_{3}=v_{c} & v_{4}=0 \\
i_{1}=i_{l} & i_{2}=0 & i_{3}=0 & i_{4}=i_{l} .
\end{array}
$$
exister.
- Phases (1)-(2)-(3). - Cette phase ne peut avoir lieu que si $v_{c}=0$. Mais alors, $i_{c}>0$ entraîne $\mathrm{d} v_{c} / \mathrm{d} t>0$ et donc $v_{c}$ augmente. Le thyristor 1 se bloque. On revient à la phase (2)-(3).

- Phase (2)-(3)-(4). - Pour des raisons analogues $\rightarrow$ (2)-(3).

- Phase (1)-(2)-(4). - Pour des raisons analogues $\rightarrow$ (1)-(4).

- Phase (1)-(3)-(4). - Pour des raisons analogues $\rightarrow$ (1)-(4)

- Phase (1)-(2)-(3)-(4). - Cette phase ne peut avoir lieu que si $v_{c}=E=0$. C'est alors l'équivalent d'une phase de roue libre.

1.2 Diagramme Des transitions. - La figure 7 reprend toutes les phases étudiées et indique les transitions possibles entre celles-ci, dans le seul cas $E>0$. Les transitions sont indiquées par des flèches portant les conditions nécessaires à leur existence. Les cercles bouclés sur une phase indiquent les conditions dans lesquelles on ne peut provoquer de transitions.

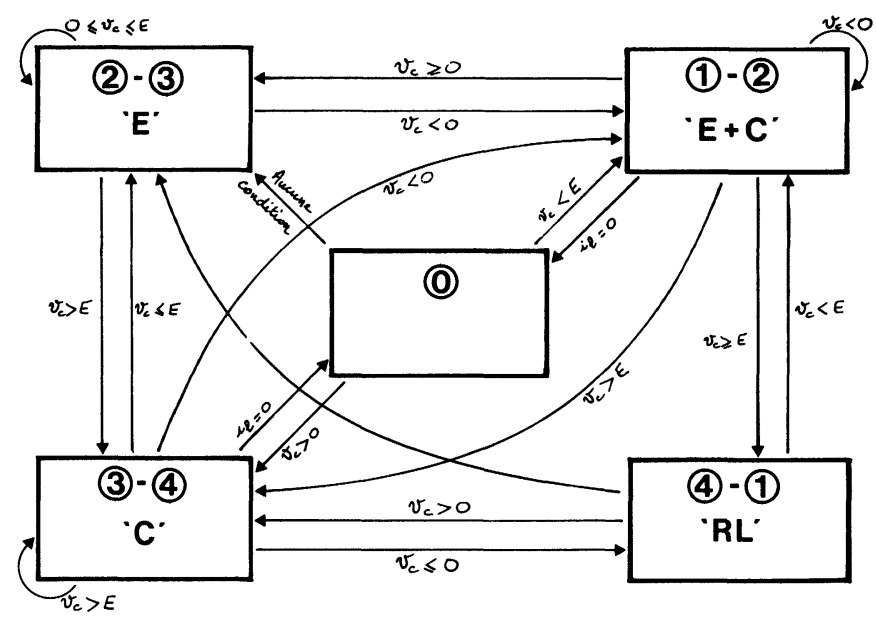

Fig. 7. - Diagramme des transitions.

[Diagram of transitions.]

1.3 EQUATIONS NORMALISÉES DE FONCTIONNEMENT. - L'examen des équations de fonctionnement montre que toutes les phases peuvent répondre au groupe de deux équations :

$$
\begin{aligned}
\frac{\mathrm{d} \varphi}{\mathrm{d} t} & =-\alpha v_{c}+\beta . E-r i_{l} \\
C \cdot \frac{\mathrm{d} v_{c}}{\mathrm{~d} t} & =\alpha . i_{l}
\end{aligned}
$$

où $\alpha$ et $\beta$ sont les coefficients pouvant prendre les valeurs 0,1 et -1 .

On peut faire apparaitre le courant $i_{l}$ à partir du flux : $\varphi=l . i_{l}$. En tenant compte du caractère non 
linéaire de l'inductance $l$ dans le cas de la machine à réluctance variable :

$$
\frac{\mathrm{d} \varphi}{\mathrm{d} t}=l \cdot \frac{\mathrm{d} i_{l}}{\mathrm{~d} t}+i_{l} \cdot \frac{\mathrm{d} l}{\mathrm{~d} t}
$$

Physiquement, les coefficients $\alpha$ et $\beta$ indiquent :

$0:$ Le condensateur est déconnecté.

$1:$ Le condensateur est connecté

$$
\begin{gathered}
\alpha\left\{\begin{array}{c}
0: \text { Le condensateur est déconnecté. } \\
1: \text { Le condensateur est connecté } \\
\text { le courant } i_{l} \text { fait croître } v_{c} ; \\
v_{c}>0 \text { fait diminuer } i_{l} . \\
-1: \text { Le condensateur est connecté; } \\
\text { le courant } i_{l} \text { fait décroître } v_{c} ; \\
v_{c}>0 \text { fait augmenter } i_{l} .
\end{array}\right. \\
\beta \begin{cases}0: \text { La source est déconnectée. } \\
1: \text { La source est connectée. }\end{cases}
\end{gathered}
$$

Pour écrire un programme de simulation, il est intéressant de normaliser les grandeurs. Nous avons effectué celle-ci par rapport à la tension d'alimen-

\begin{tabular}{|c|c|c|c|}
\hline Graudeure Puysique & Graudeune Resue & GRANDEUR REDUite & G. de Normalisation \\
\hline TEmps & t & $\tau$ & $T_{e}$ \\
\hline TENSION & $v$ & $\nu$ & E \\
\hline Courant & $i$ & 1 & $C E / T_{e}$ \\
\hline IndUCTANCE & $\ell$ & $\lambda$ & $T_{e}^{2} / C$ \\
\hline Flux & $\varphi$ & $\psi$ & $E T_{e}$ \\
\hline Resistance & r & $p$ & $T_{e} / C$ \\
\hline
\end{tabular}
tation $E$, à la période électrique de fonctionnement $T_{\mathrm{e}}$ et au condensateur $C$. (Tableau de la figure 8.)

Fig. 8. - Tableau de normalisation.

[Table of normalisation.]

Les équations peuvent alors s'écrire :

$$
\begin{aligned}
& \frac{\mathrm{d} j}{\mathrm{~d} \tau}=-\frac{(\mathrm{d} \lambda / \mathrm{d} \tau)}{\lambda} \cdot j-\frac{\alpha}{\lambda} \cdot v+\frac{\beta}{\lambda}-\frac{\rho}{\lambda} \cdot j \\
& \frac{\mathrm{d} v}{\mathrm{~d} \tau}=\alpha . j .
\end{aligned}
$$

Un programme numérique de simulation a été écrit pour résoudre ce jeu d'équations [2]. La donnée des paramètres $\alpha$ et $\beta$ suivant le tableau de la figure 9 permet de résoudre successivement toutes les phases d'un cycle de fonctionnement et de prédéterminer ainsi un régime permanent.

\begin{tabular}{|c|c|c|}
\hline Phase & $\alpha$ & $\beta$ \\
\hline (0) & 0 & 0 \\
(1) - (2) & 1 & 1 \\
(2) - (3) & 0 & 1 \\
(3) - (4) & -1 & 0 \\
(1) - (4) & 0 & 0 \\
\hline
\end{tabular}

Fig. 9. - Coefficients des équations généralisées. [Coefficients of the generalised equations.]
2. Etude des cycles de fonctionnement. - Il s'agit maintenant de rechercher tous les enchaînements possibles de phases. Ces enchaînements sont appelés cycles.

Dans une structure à thyristors, il est absolument indispensable de se préoccuper du temps de polarisation inverse des thyristors $\left(t_{\mathrm{q}}\right)$. Si pendant les phases (1)-(4) et (2)-(3), la tension inverse aux bornes du thyristor qui vient d'être bloqué reste constante pendant toute la durée de la phase, par contre, dans les phases (1)-(2) et (3)-(4), la présence du condensateur $C$ amène une variation de la tension réappliquée au thyristor. En ce qui concerne les transitions à partir et vers la phase (0), aucun problème particulier ne se pose.

2.1 ReCHERCHE DES CYCLES PERMIS. - Dans une transition d'une phase à une autre, il est nécessaire de vérifier la continuité de la tension aux bornes du condensateur $\left(v_{c}\right)$ et du courant dans le bobinage $\left(i_{l}\right)$. Pour connaitre les cycles permis, il nous faut reprendre le diagramme des transitions (Fig. 7) où chaque phase va être divisée en trois sous-phases suivant la tension $v_{c}$ :

- $v_{c}$ est compris entre 0 et $E$,

- $v_{c}$ est au-dessus de cet intervalle,

$-v_{c}$ est en dessous de cet intervalle.

Dans les diagrammes que l'on peut alors tracer, il apparaît trois sortes de sous-phases :

- des sous-phases normales;

- des sous-phases où l'on ne peut arriver (fantômes). Par exemple : (1)-(4) avec $0<v_{c}<E$;

- des sous-phases d'où l'on ne peut repartir (pièges). Par exemple, (2)-(3) avec $0<v_{c}<E$. Ces dernières sont dangereuses pour le fonctionnement de l'ensemble puisque l'on perd alors toute faculté d'agir sur l'ensemble convertisseur-charge.

On peut alors redessiner uniquement l'ensemble des sous-phases normales et les transitions possibles y affèrent (Fig. 10). On a supposé, pour tracer ces diagrammes, que le circuit $l, C$ était peu amorti (la phase (1)-(4) ne retombe pas à (0). D'autre part, les cas limites ne sont pas représentés. Il est absolument impossible de rester sur une frontière. Les courants de fuite (condensateur et thyristors) font évoluer $v_{c}$ d'une manière totalement aléatoire, déterminant l'une ou l'autre des sous-phases de part et d'autre de la frontière. Pour cette raison, il est tout à fait déconseillé de rester trop près de ces frontières.

Nous avons d'autre part fait apparaitre la tension appliquée au thyristor qui vient d'être bloqué. On notera que :

- cette tension reste fixe pendant les phases (1)-(4) et (2)-(3) mais évolue pendant les phases (1)-(2) et (3)-(4),

- les tensions négatives réappliquées sur le thyristors sont plus importantes quand on sort d'une phase (1)-(4) que quand on sort d'une phase (2)-(3) ce qui est plus intéressant pour blocage, 


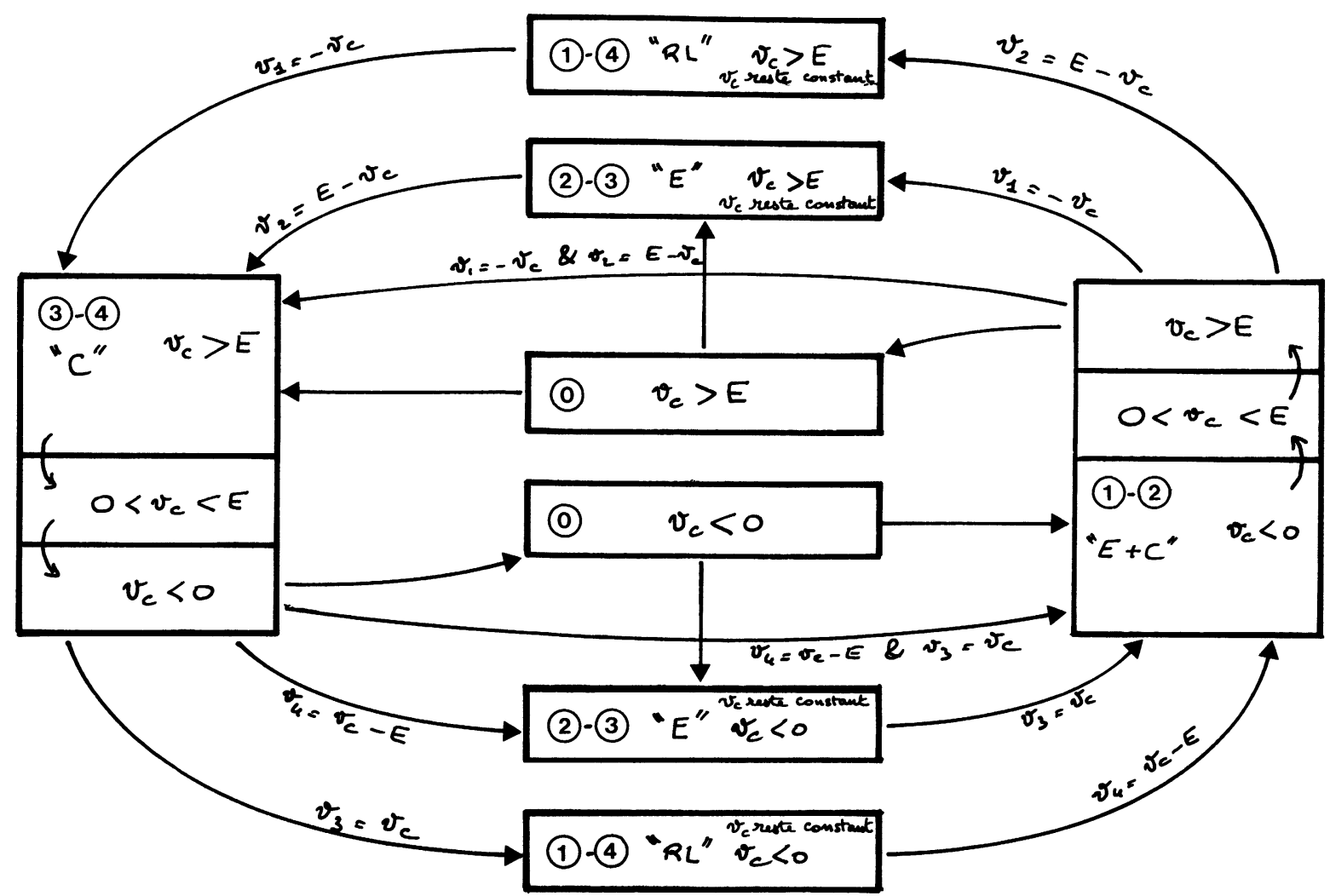

Fig. 10. - Diagramme récapitulatif des cycles.

[Recapitulatory diagram of the cycles.]

- une phase $" E+C$ » permet d'atteindre plus vite un courant important qu'une phase $" C$ » si $E>0$.

2.2 RECHERCHE DES CYCLES INTÉRESSANTS. - Un cycle ne peut être qualifié d'intéressant que vis-à-vis d'une charge bien définie. La charge la plus complexe que nous ayons étudiée est la machine à réluctance variable (M.R.V.). Aussi faut-il rappeler quelques propriétés essentielles la concernant.

Vue des bornes de son enroulement supposé unique, la M.R.V. se comporte comme une inductance variable. On montre [4] que le couple électromagnétique instantané $c$ produit sur l'arbre est en régime non saturé :

$$
c=\frac{1}{2} \cdot i_{l}^{2} \cdot \frac{\mathrm{d} l}{\mathrm{~d} \theta_{\mathrm{m}}}=\frac{P}{2} \cdot i_{l}^{2} \cdot \frac{\mathrm{d} l}{\mathrm{~d} \theta_{\mathrm{e}}}
$$

où : $i_{l}$ est le courant instantané dans l'enroulement, $\mathrm{d} l / \mathrm{d} \theta$ est la dérivée de l'inductance $l$ de l'enroulement par rapport à $\theta, \theta_{\mathrm{m}}$ est l'angle mécanique de rotation, $P$ est le nombre de plots de la machine, $\theta_{\mathrm{e}}=P \cdot \theta_{\mathrm{m}}$ est l'angle électrique de la machine.

Pour obtenir un couple moyen moteur maximal et minimiser le couple pulsatoire, il est intéressant de travailler à courant nul quand $\mathrm{d} l / \mathrm{d} \theta<0$. Les cycles de fonctionnement répondant à cette condition comprendront nécessairement une phase (0).

Comme nous l'avions mentionné dans l'introduc- tion, le convertisseur avait été conçu pour fournir une forme d'onde la plus proche possible du créneau de courant. Les cycles principaux de fonctionnement comprendront donc deux phases de transfert d'énergie (du condensateur vers le bobinage et vice versa) pour les montées et descentes rapides du courant et entre les deux une phase où le courant ne variera pas trop (phases d'alimentation ou de roue libre). A mesure que la fréquence augmente, les durées des phases de montée et de descente du courant étant sensiblement invariantes (circuit $l C$ ), la durée de la phase intermédiaire s'amenuise et peut éventuellement être nulle. Les six possibilités décrites ci-dessus sont rassemblées sur la figure 11.

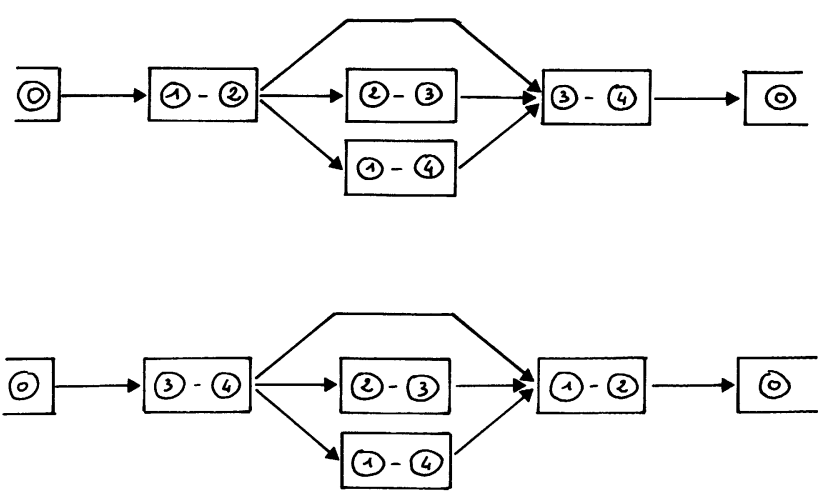

Fig. 11 . 
Il est possible de tirer d'autres cycles de la figure 10 . Il y a d'abord les cycles :

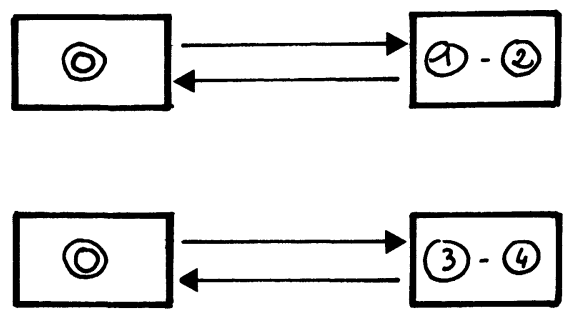

Fig. 12.

dont le rôle principal est d'inverser la tension aux bornes du condensateur. On peut les utiliser pour passer d'un groupe à l'autre des cycles de la figure 11 .

Le premier de ces cycles permet de plus un apport d'énergie de la source dans le condensateur. Dans ces deux cycles, l'extinction est naturelle.

Si l'on désire un courant ininterrompu dans la charge, on peut utiliser le cycle :

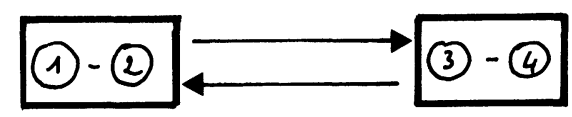

Fig. 13.

Il existe d'autres cycles que nous ne développerons pas car ils ne présentent que peu d'intérêt ou sont des associations des précédents.

Il est maintenant nécessaire de vérifier la polarisation inverse des thyristors. Examinons le problème sur le premier groupe de cycle de la figure 10. Ces cycles ne peuvent exister que si $v_{c}<0$ pendant la phase (0) (voir Fig. 10). Par amorçage des thyristors 1 et 2 , la tension croît et devient supérieure à $E$. Il est alors parfaitement possible de passer en phase (2)-(3), la polarisation négative du thyristor 1 est effective $(<-E)$ et dure tant que dure la phase (2)-(3). Par amorçage du thyristor 4, on peut passer en phase (3)-(4). Le temps de polarisation inverse du thyristor 2 est une fonction du courant $i_{l}$ au moment de l'amorçage du thyristor 4 , de la valeur du condensateur $C$ et de la tension $v_{c}$ au moment du passage de la phase (1)-(2) à la phase (2)-(3). Il apparaît ici une fonction importante de la commande qui doit prévoir le déroulement des phases avant d'envoyer un ordre de déblocage à un thyristor. En l'occurrence, la commande doit vérifier au moment de l'amorçage du thyristor 3 si la tension $v_{c}$ est suffisamment négative pour, plus tard, pouvoir amorcer le thyristor 4 sans risque de voir se réamorcer le thyristor 2 . Il en est de même pour les autres cycles. Une première commande numérique câblée qui ne prenait en compte qu'un petit nombre de cycles a fait place à une commande par microprocesseur [5].
3. Résultats expérimentaux. - Tous les cycles de fonctionnement décrits dans le paragraphe précédent ont été obtenus sur un convertisseur de puissance alimentant une machine à réluctance variable ou un électro-aimant. Nous présentons ci-après une sélection des cycles les plus intéressants parmi ceux obtenus sur la M.R.V. du laboratoire d'Electrotechnique de la Faculté des Sciences d'Orsay [6], [7]. Les choix du cycle et des paramètres de fonctionnement se font actuellement par un clavier de commande géré par le microprocesseur. Le réglage du couple moteur (et donc de la vitesse) est obtenu par modification des angles de décalage entre la position du rotor et le déclenchement des diverses phases de fonctionnement, ainsi que sur la tension d'alimentation $E$. Tous les essais présentés sont obtenus en régime établi. Les problèmes de passage automatique entre les diverses phases de fonctionnement n'ont pas encore été étudiés et feront l'objet de travaux à venir.

Dans les expérimentations qui suivent, l'inductance moyenne vue par le convertisseur est $1 \mathrm{mH}$; la capacité du condensateur est $84 \mu \mathrm{F}$.

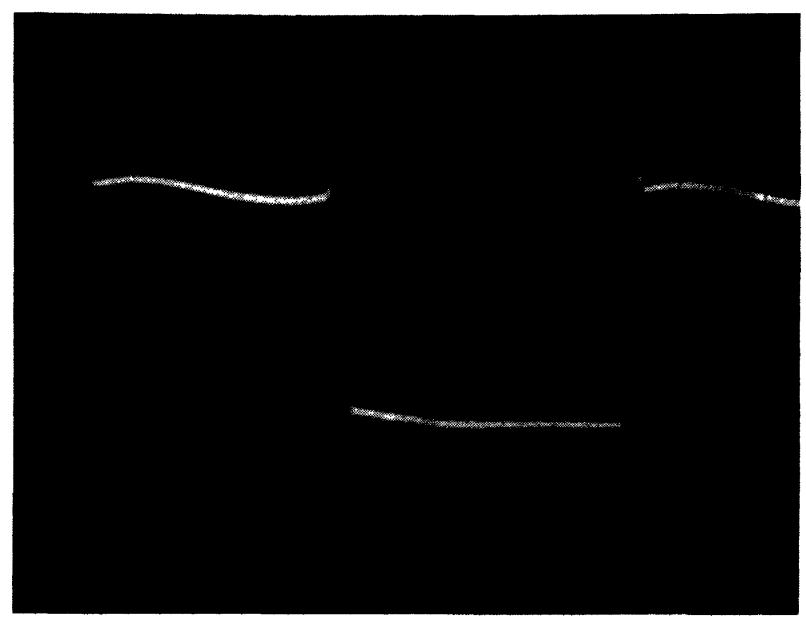

Fig. 14. - Courant dans la machine $50 \mathrm{~A} / \mathrm{div} 2 \mathrm{~ms} / \mathrm{div}$.

[Motor current $50 \mathrm{~A} / \mathrm{div} 2 \mathrm{~ms} / \mathrm{div}$.]

La fréquence de fonctionnement est $78 \mathrm{~Hz}$. Les temps de montée et de descente sont de l'ordre de $500 \mu \mathrm{s}$, le rapport cyclique, réglable, est proche de $1 / 2$. Le plateau de courant se situe à $130 \mathrm{~A}$ environ. Les petits dépassements sur le courant sont dus à la nécessité de polariser en inverse le thyristor que l'on vient de bloquer.

La figure 15 montre un cycle (0) $\rightarrow$ (1)-(2) $\rightarrow$ (2)-(3) $\rightarrow$ (3)-(4) $\rightarrow$ (2) à une fréquence supérieure $(170 \mathrm{~Hz})$ à celle du fonctionnement précédent. Les temps de montée et de descente sont inchangés.

Quand la fréquence augmente encore, il peut être difficile de placer une phase d'alimentation pure (2)-(3). On peut alors passer directement de la phase (3)-(4) à la phase (1)-(2) ou vice versa. La figure 16 illustre un cycle (1) $\rightarrow$ (3)-(4) $\rightarrow$ (1)-(2) $\rightarrow$ (1) où l'effet de 


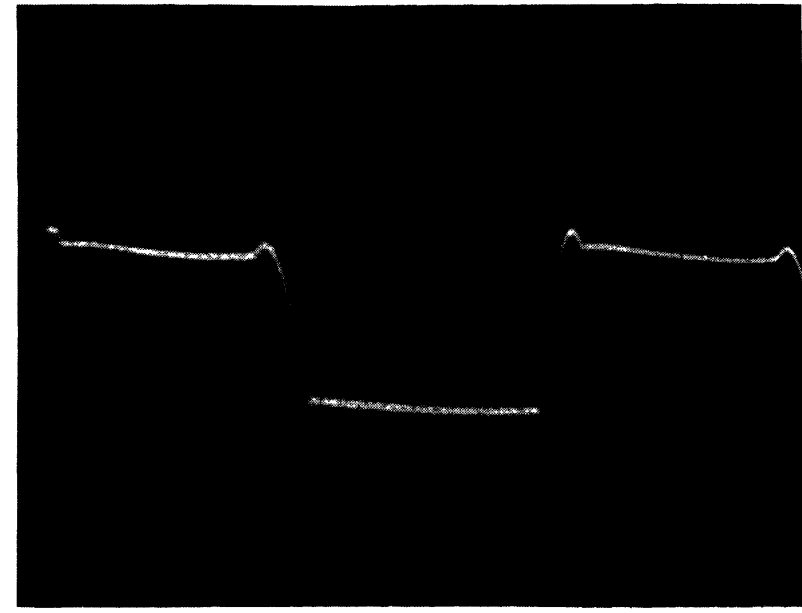

Fig. 15. - Courant dans la machine $50 \mathrm{~A} / \mathrm{div} 1 \mathrm{~ms} / \mathrm{div}$. [Motor current $50 \mathrm{~A} / \mathrm{div} 1 \mathrm{~ms} / \mathrm{div}$.]

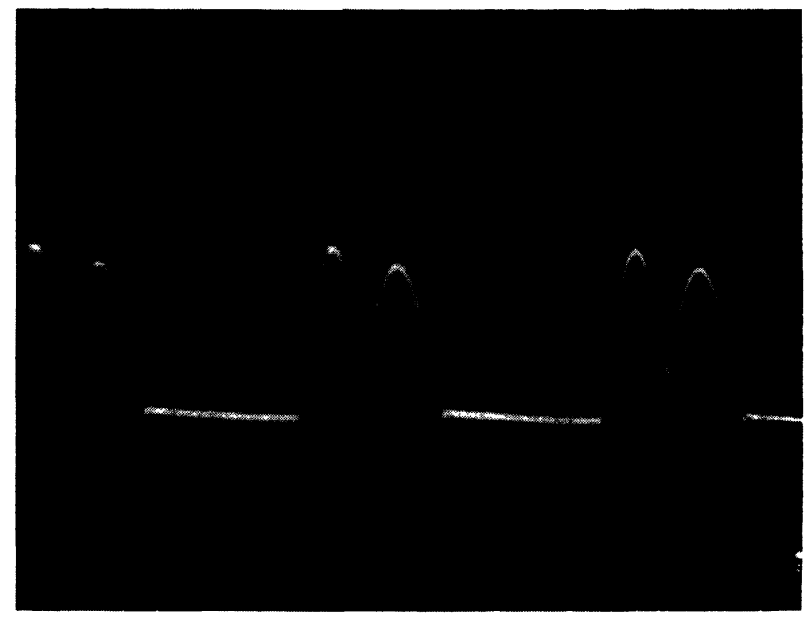

Fig. 16. - Courant dans la machine $50 \mathrm{~A} / \mathrm{div} 1 \mathrm{~ms} / \mathrm{div}$.

[Motor current $50 \mathrm{~A} / \mathrm{div} 1 \mathrm{~ms} / \mathrm{div}$.]

creux a été volontairement exagéré. La fréquence est $290 \mathrm{~Hz}$.

Quand la fréquence augmente encore, on a intérêt à créer des ondes quasi semi-sinusoïdales. Le courant représenté figure 17 est dû à une succession des phases (1)-(2), (0), (3)-(4) et (0) à $420 \mathrm{~Hz}$.

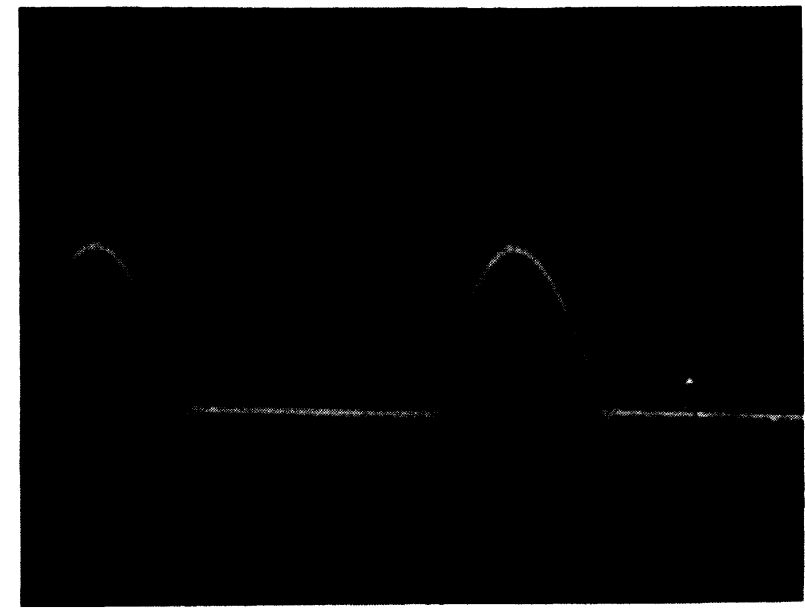

Fig. 17. - Courant dans la machine $50 \mathrm{~A} / \mathrm{div} 500 \mu \mathrm{s} / \mathrm{div}$.

[Motor current $50 \mathrm{~A} / \mathrm{div} 500 \mu \mathrm{s} / \mathrm{div}$.]

En modifiant l'enroulement de la machine et en diminuant la capacité du condensateur, il a été possible de dépasser la fréquence de $1000 \mathrm{~Hz}$ avec le fonctionnement précédent.

4. Conclusion. - Une étude poussée du fonctionnement d'un convertisseur a permis la définition d'une commande améliorant très nettement les performances de l'ensemble convertisseur-charge. Il a été alors possible de faire fonctionner une machine à réluctance en vitesse variable avec une fréquence d'alimentation variant entre 0 et plus de $1000 \mathrm{~Hz}$, le courant dans l'enroulement dépassant $100 \mathrm{~A}$. Cela a été rendu possible par une étude systématique de toutes les phases conduisant à un fonctionnement problématique. L'implantation d'un microprocesseur dans la commande a permis de prendre en compte tous ces phénomènes. Il autorise en effet la modification des cycles de fonctionnement la machine étant en rotation.

Remerciements. - Nous remercions Monsieur Rioux du laboratoire d'Electrotechnique d'Orsay, chez qui nous avons pu tester le convertisseur décrit ci-dessus grâce à une machine de puissance notable. 


\section{Bibliographie}

[1] Nuta, J., Bleiss, C. A., Glaize, C. and Goyet, R., Tests of an axial air-gap variable reluctace motor supplied by a solid-state converter. Electric Machines and Electromecanics. Vol. 6, no 4.

[2] Allano, S., Simulation de convertisseur d'alimentation de machines à réluctance variable. Commande en logique programmée. D.E.A. Juin 80. E.N.S.E.T. Cachan.

[3] Glaize, C., Etude systématique de la commande d'une structure d'alimentation en courant de systèmes magnétiques inductifs. Rapport interne EA/ 10.80/CG. E.N.S.E.T. Cachan.

[4] UnNewehr, L. E., KoCH, W. H., An axial air-gap reluctance motor for variable speed application.
IEEE Trans. on Power App. and Syst. PAS 93 (1974) 367-374.

[5] Glaize, C., Stromboni, J. P., Carte de commande universelle à microprocesseur. Application au contrôle d'un variateur de vitesse. CONUMEL 80. Lyon pp. II-31 à II-40.

[6] Goyet, R. Bleiss, C. A. et Rioux, C., Expérimentation d'un prototype d'essai de machine à réluctance variable à disques imbriqués. Revue Phys. Appl. 15 (1980) 1451-1458.

[7] Goyet, R. and Bleiss, C. A., Experimental study of an elementary interleaved disk reluctance motor. I.C.E.M. 80 Athenes. pp. 427-434. 